\title{
Assessment of Piriformis Tightness in Third Trimester of Pregnancy - A Prevalence Study
}

\author{
Priyanka Rathod $^{1}$, Payal Dhawale ${ }^{2}$, Goutami Katage ${ }^{3}$ \\ ${ }^{1}$ B.P.Th, MGM Institute of Physiotherapy, Aurangabad. \\ ${ }^{2}$ M.P.Th SPORTS, Assistant Professor, MGM Institute of Physiotherapy, Aurangabad. \\ ${ }^{3}$ M.P.Th Musculoskeletal, Assistant Professor, MGM Institute of Physiotherapy, Aurangabad. \\ Corresponding Author: Priyanka Rathod
}

DOI: https://doi.org/10.52403/ijhsr.20220309

\begin{abstract}
Background: Gestational pain in the lower back region is a common concern, affecting up to $50 \%$ of pregnant women at some point in their gestation. Prolonged sitting position, biomechanical changes during pregnancy are the foremost cause of piriformis tightness in female that may eventually leads to piriformis syndrome and low back pain. Therefore, the present study was designed to identify the prevalence of piriformis tightness in pregnant females.

Methods: 93 subjects enrolled in this study were evaluated and screened on the basis of the inclusion and exclusion criteria. Tightness of piriformis muscle was assessed using piriformis test $\&$ the limited ROM of hip due to tightness of piriformis muscle by using goniometer.

Results: Data was analyzed using graph pad prism software. Piriformis tightness was found to be present in 27 subjects (29\%). Prevalence of piriformis tightness was less in third trimester of pregnancy. However, the prevalence of piriformis tightness in females was statistically significant.

Conclusion: Prevalence of piriformis muscle tightness in pregnant women of third trimester was very less in this study which concludes that females who were in third trimester of pregnancy not having piriformis tightness because of relaxin hormone which relaxes all the muscle for the normal growth of fetus.
\end{abstract}

Key Words: Gestational low back pain, Piriformis muscle tightness, Piriformis syndrome, Pregnancy, Third trimester.

\section{INTRODUCTION}

Pregnancy is a physiological condition with reversible hemodynamic, neurohormonal \& coagulation changes to the maternal body during 9 month period ${ }^{(1)}$. Pregnant women undergo profound anatomical and physiological changes so that they can cope with the increased physical and metabolic demands of their pregnancies $^{(2)}$. The cardiovascular, respiratory, haematological, renal, gastro intestinal and endocrine systems all undergo important physiological alteration and adaptations needed to allow development of the fetus and to allow the mother and fetus to survive the demands of childbirth ${ }^{(2)}$.
Pregnancy induced biomechanical, hormonal and vascular changes are likely to give rise to wide variety of musculoskeletal problem $^{(2)}$. Joint laxity develops secondary to hormone level fluctuation. Fluid retention leads to compression of soft tissues in pregnancy. spinal pain has been reported as the most frequent disorder ${ }^{(2)}$. One of the most frequent complication of pregnancy is low back pain and its incidence is higher in third trimester of pregnancy, when the most important biomechanical and morphological changes take place ${ }^{(2)}$.

From the second trimester, abdominal morphology is altered by the increased size of the uterus and the 
heaviness of the fetus, with a $30 \%$ gain in abdominal mass ${ }^{(2)}$. The increased size of the abdomen has been linked to a decreased static stability and adaptive changes in spinal curvatures, which would compensate the anterior displacement of center of gravity to ensure postural balance (2). Postural alterations most frequently are increase lumbar curvature, pelvic ante version, increased thoracic curvature, increased cervical curvature, protraction of shoulder girdle, hyper-extended knees, extension of ankles ${ }^{(2)}$. As the pregnancy progresses, the lower trunk segment inertial characteristics show a significantly larger rate of increase than any other body segments, with the rapid changes in mass, \& moment of inertia trunk segment kinematics may be altered in daily activities such as walking $^{(2)}$. Due to adaptive changes in static posture the powerful changes are also distinctive and include shortened hip flexors, lower back musculature, and pectorals. Abdominal muscles, neck, \& upper back muscle groups elongate which may promote stretch weakness ${ }^{(2)}$. In bones and joint there is a tendency to decalcification of bones, subluxation of joints due to softening of ligaments by relaxing hormone, which is more marked in sacroiliac joint \&symphysis pubis, which allows stretching at the time of delivery ${ }^{(2)}$. Kinematic gait parameters suggests an increased role of hip abductors, hip extensor and ankle plantar flexors muscle groups which can leads to development of waddling gate pattern ${ }^{(2)}$.

Third trimester of pregnancy is from $27^{\text {th }}$ week to end of pregnancy (3). Gestational low back pain is a major complaint during gestation, being considered a multifactorial symptom affecting the lumbar region which may irradiate to lower limbs. The patient's pain increased after prolonged sitting, walking, and climbing stairs ${ }^{(4)}$. Herniation or bulging of an intervertebral disc, causing nerve compression $^{(5)}$. Other possible causes would be posture changes, pelvic insufficiency and direct pressure of the fetus and gravid uterus on nervous roots of the lumbosacral spine. Added to these factors, previous to gestation is also a major risk factor for gestational low back pain ${ }^{(6)}$. Low back pain (LBP) is the most disabling condition worldwide and one of the most common causes of LBP is piriformis syndrome that results from tightness of piriformis muscle. Reported incidence rates for piriformis syndrome among patients with low back pain vary widely from $5 \%$ to $36 \% .{ }^{(7)}$. Approximately $50 \%$ of pregnant women have low back pain during gestational.

Piriformis muscle originates from pelvic surface of the sacrum between (and lateral to) the first through fourth pelvic sacral foramina, margin of the greater sciatic foramen and pelvic surface of the sacrotuberous ligament and inserted to the superior border of the greater trochanter of the femur via a round tendon that, in many individuals, is merged with the tendons of the obturator internus and gemelli muscles $\&$ it is supplied by sciatic nerve. The piriformis muscle acts as an external rotator, weak abductor and weak flexor of the hip, providing postural stability during ambulation and standing. Piriformis muscle acts as an external rotator when angle of hip flexion is $60^{\circ}$ or less and the function changes when angle of hip flexion is greater than $60^{\circ}$, so that it becomes an internal rotator of the hip. From this it could be stated that piriformis remains active during any type of sitting position whether high sitting or cross sitting although its function changes in different styles of sitting. ${ }^{(7)}$

Piriformis muscle has a predominance of type-I fibers which has a tendency to develop shortness or tightness when the muscle is abnormally stressed. When the piriformis is shortened its diameter increases and because of its location it creates pressure to the sciatic nerve which passes under it in $80 \%$ of population. The perpetual loading of the piriformis muscle through over lengthening and eccentric demand during functional activities secondary to weak agonist 
muscles may result in sciatic nerve compression or irritation. ${ }^{(7)}$.

Compressive Damage to the Proximal Sciatic Nerve by the Piriformis Muscle $^{(8)}$. When the piriformis becomes tight it can put pressure on the sciatic nerve causing irritation and sending pain down the back of the leg (sciatica). Peripheral neuritis of the sciatic nerve caused by pressure of an injured or irritated piriformis muscle or an abnormal condition of the piriformis muscle lead to piriformis syndrome $^{(9)}$.

In most cases of piriformis syndrome occurs due to contracted piriformis muscle, the sacrum anteriorly rotated toward the ipsilateral side on a contralateral oblique axis, resulting in compensatory rotation of the lower lumbar vertebrae in the opposite direction, this facilitation and compensatory somatic dysfunctions may lead to low back pain, thoracic and cervical pains. Delayed diagnosis of piriformis syndrome may lead to pathologic conditions of the sciatic nerve, chronic somatic dysfunction and compensatory changes and it may cause pain, paresthesia, hyperesthesia and muscle weakness. In extreme cases, misdiagnosis of piriformis syndrome-related back pain with "sciatica" as prolapsed intervertebral disc may lead to unnecessary surgery ${ }^{(8)}$. As the action of piriformis is external rotation \& abduction, the internal rotation \& adduction are limited respectively. ${ }^{(10)}$ As the mobility of pregnant women reduce in third trimester of pregnancy, due to increase in weight of both fetus\& mother, anatomical changes \& physiological changes takes place during pregnancy \& because of this muscle imbalance can occur. Therefore, the present study is design to identify prevalence of piriformis tightness in third trimester of pregnancy so that appropriate preventive and corrective measures can be taken in time which may reduce the rate of development of Low back pain because of piriformis tightness.

\section{MATERIAL \& METHODS}

The current study is an observational study, started after approval of the institutional ethical committee. Women aged between 18 to 30 years were included in the study. For the study source of women during third trimester of pregnancy from Aurangabad city was selected by convenient randomized sampling. Population of 93 pregnant women in third trimester of pregnancy, who were willing to participate in study were selected. Pregnant women with clinical complication like preeclampsia, gestational diabetes mellitus, diagnosed musculoskeletal cases of rheumatoid arthritis or osteoarthritis of the spine or hips were excluded. Aim and procedure of the study were explained to all subjects in their preferred language before data collection. Informed consent form (which also includes permission to use their data and photograph for presentation and publication purpose) written in their preferred language (English/Hindi/ Marathi) was taken from all the subjects who were agree to participate. Demographic data including age, sex and occupation was collected. Prior to the test, all the subjects underwent comfortable walk at selfdetermined pace around the research venue under therapist supervision.

1. Piriformis test

The active piriformis test had sensitivity of 0.78 , specificity of 0.80 , positive likelihood ratio of 3.90, negative likelihood ratio of 0.27 , \& diagnostic odd ratio of $14.40^{(12)}$.

2. ROM- Range of motion: Hip internal rotation and hip adduction

Measured by goniometry ${ }^{(15)}$

Piriformis tightness was check through piriformis test. In about $15 \%$ of population, the sciatic nerve, all or in part, pass through the piriformis muscle rather than below it. These people were more likely to suffer from this relatively rare condition, piriformis syndrome. The test was assessed by the assessor (batchmate). The patient was in the side lying position with the test leg uppermost, the patient 
flexes the test hip to $60^{\circ}$ with the knee flexed. The examiner stabilizes the hip with one hand and applies a downward pressure to the knee. The indifferent hand is initially used to palpate the position of the fetus to ensure that the flexed upper leg of the patient does not create pressure on the fetus. When the leg of the patient was used as a lever, the amount of flexion at the hip was decreased $^{(13)}$. If the piriformis muscle was tight, pain was elicited in the muscle. If the piriformis muscle was pinching the sciatic nerve, pain results in the buttock and sciatica may experience by the patient. Resisted lateral rotation with the muscle on stretch (hip medially rotated) can cause the same sciatica ${ }^{(14)}$. The knee of the tested side was push into adduction, to place a stretch on the piriformis muscle. If the adduction ROM was limited \& the patient reported discomfort posterior to the tested greater trochanter tested side piriformis was considered tight (positive) ${ }^{(12)}$.

As the primary action of piriformis is hip external rotation and hip abduction, the opposite action that is internal rotation and adduction will be limited. Normal range of motion of hip internal rotation \& adduction were $40^{\circ}-45^{\circ} \& \quad 40^{\circ}-45^{\circ}$ respectively. Hence due to tightness of piriformis muscle these two actions were limited, measured by goniometer ${ }^{(15)}$.

\section{STATISTICAL ANALYSIS}

The outcome measures/ parameters were filled in Microsoft office excel sheet version.2010 and were statistically analyzed. The mean scores were calculated using the scientific calculator. The values were documented in Microsoft office excel sheet version.2010. The range of motion of hip joint are collected. The P-value and Tvalue were calculated in graph-pad software version.2018 and then filled in Microsoft office excel sheet v.2010.

\section{RESULT}

Prevalence of piriformis muscle tightness was checked among 93 subjects with an age range of 18-30 years. We divided age group into 2 subgroup that is $18-24 \& 25-30$ years. In present study no of participant in first age group were $77 \%$ \& in second group $21 \%$ which is shown in table number $1 \&$ percentage in graph number 1 . According to the BMI classification 53\% of subjects were from normal BMI, $41 \%$ from overweight category \& $6 \%$ from obese class 1 category which is shown in table number $2 \&$ graph number 2 . Out of 93 subjects, 27 subjects were clinically diagnosed with piriformis tightness \& 66 subjects had no tightness. This overall prevalence of the piriformis tightness was found to be statistically significant which is shown in table 3 \& graph number 3 . When we compared of ROM i.e. internal rotation and adduction of both right and left side in Positive and Negative Subjects of Third Trimester of pregnancy, we found mean \pm SD for right \& left hip internal rotation was $36.04 \pm 3.156 \& 36.11 \pm 3.191$ respectively in positive participants. While in negative participants the mean \pm SD for right \& left hip internal rotation was $39.82 \pm 2.997 \&$ $39.58 \pm 2.925$ respectively whereas for hip adduction the mean \pm SD for right \& left was $38.19 \pm 2.149 \& 40.09 \pm 1.998$ respectively in positive participants . While in negative participants the mean \pm SD for right \& left hip adduction was $37.74 \pm 2.246 \& 39.82 \pm$ 2.320 respectively. The p-value was $<0.0001 \&$ t-value was 5.050 .

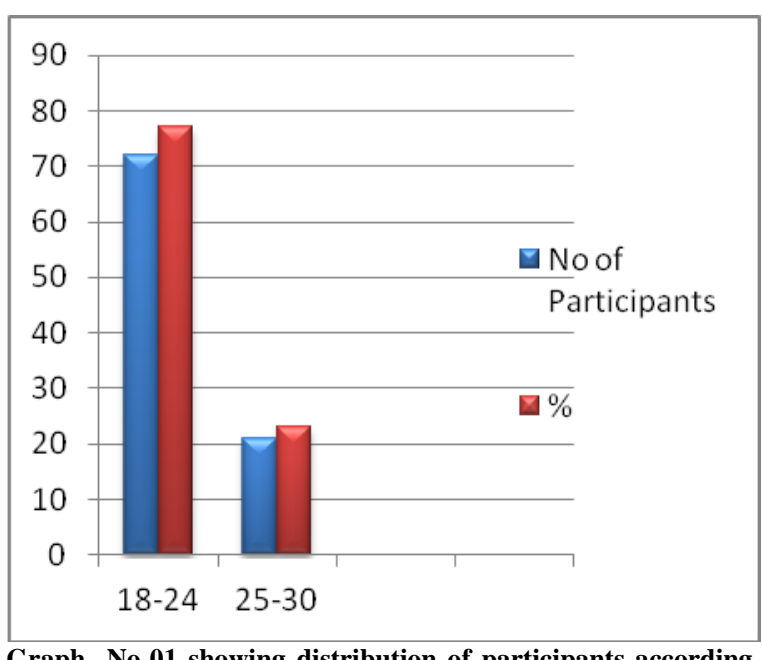

Graph No-01 showing distribution of participants according to age group. 
Priyanka Rathod et.al. Assessment of piriformis tightness in third trimester of pregnancy-a prevalence study.

Table No- 1 Baseline Characteristics

\begin{tabular}{|l|l|l|l|l|l|}
\hline & \multicolumn{2}{|c|}{ Age Group } & \multicolumn{3}{c|}{ BMI } \\
\hline & $\mathbf{1 8 - 2 4}$ & $\mathbf{2 5 - 3 0}$ & Normal & obese & Obese class 1 \\
\hline No. of participants & 72 & 21 & 49 & 38 & 6 \\
\hline Percentage & 77 & 23 & 53 & 41 & 6 \\
\hline
\end{tabular}

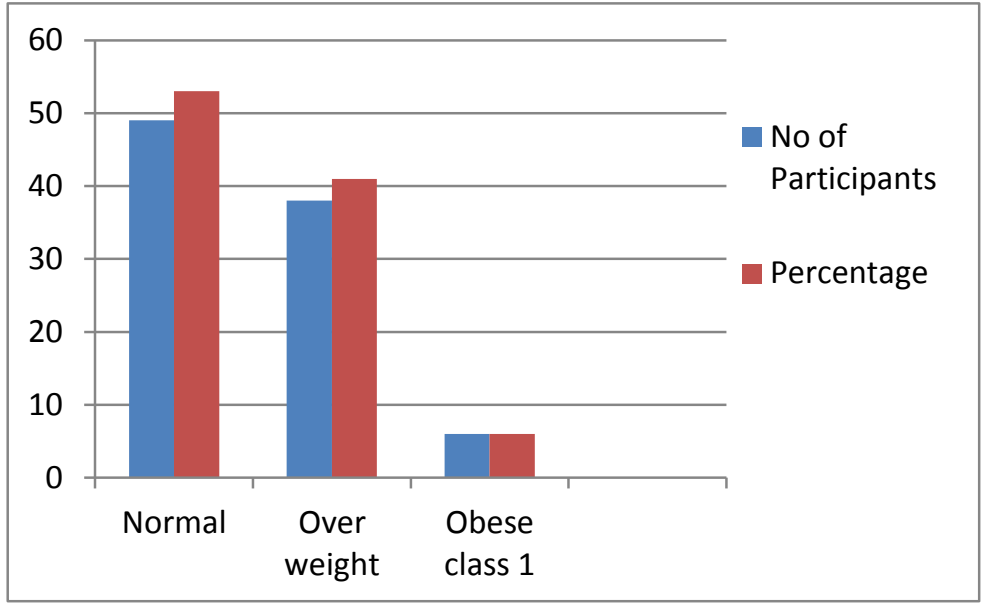

Graph No - 2 showing distribution of participants according to BMI.

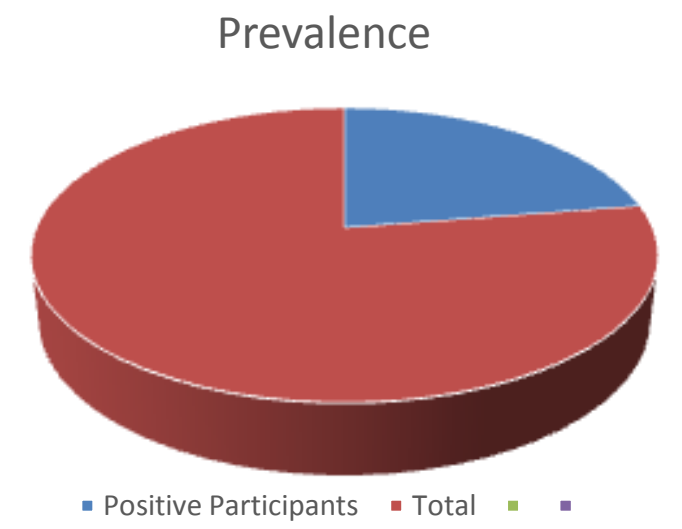

Graph no -3 showing distribution of participants according to BMI.

\begin{tabular}{|l|l|l|l|l|l|}
\hline \multirow{2}{*}{ ROM } & Side & $\begin{array}{l}\text { Positive } \\
\text { Mean } \pm \text { SD }\end{array}$ & $\begin{array}{l}\text { Negative } \\
\text { Mean } \pm \text { SD }\end{array}$ & t-value & p-value \\
\hline \multirow{2}{*}{ Internal rotation } & Right & $36.04 \pm 3.156$ & $39.82 \pm 2.997$ & 5.439 & $<0.0001$ \\
\cline { 2 - 6 } & Left & $36.11 \pm 3.191$ & $39.58 \pm 2.925$ & 5.05 & $<0.0001$ \\
\hline \multirow{2}{*}{ External rotation } & Right & $38.19 \pm 2.149$ & $40.09 \pm 1.998$ & 4.085 & $<0.0001$ \\
\cline { 2 - 6 } & Left & $37.74 \pm 2.246$ & $39.82 \pm 2.320$ & 3.955 & 0.0002 \\
\hline \multirow{2}{*}{ Piriformis test +ve } & Prevalence & Percentage & & \\
\cline { 2 - 6 } & 27 & $29 \%$ & \\
\hline
\end{tabular}

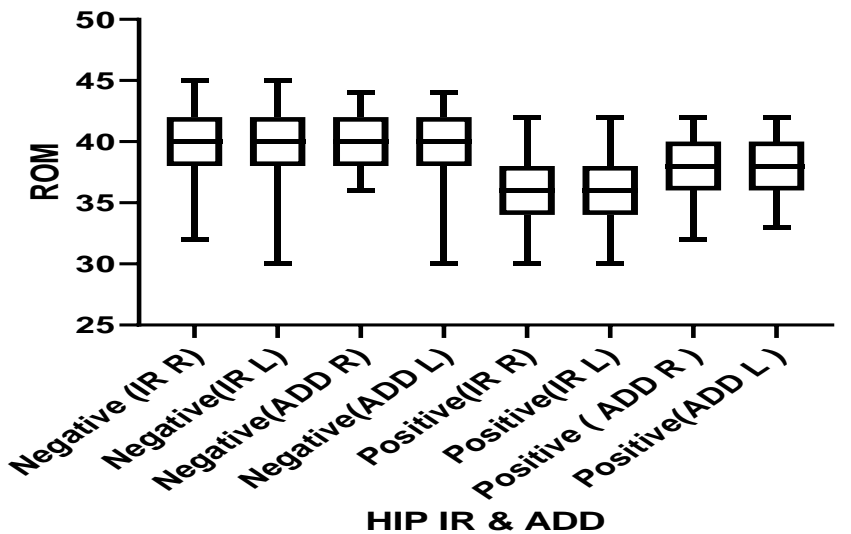


Table No 04 showing mean values of positive and negative piriformis test of participants and Comparison of ROM i.e. internal rotation and adduction of both right and left side in Positive and Negative Subjects of Third Trimester of pregnancy.

\section{DISCUSSION}

In the present study, the assessment of piriformis muscle in third trimester of pregnancy was conducted by using piriformis test which is special test to check the tightness of piriformis muscle with the sensitivity of 0.78 , specificity of 0.80 , positive likelihood ratio of 3.90 , negative likelihood ratio of 0.27 , \& diagnostic odd ratio of $14.40^{(12)}$. Through this test we got to know tightness of muscle \& reduced ROM of hip internal rotation \& hip adduction. So the present study was designed and conducted with a goal to find out the prevalence of piriformis tightness in pregnant women.

Prolong sitting may develop tightness of back and hip muscles such as hamstrings, iliopsoas, piriformis etc and create strain on joints which remains in a constant position for long time, especially when the muscles are under constant lowgrade contraction in order to hold and maintain the sitting position ${ }^{16}$. When the piriformis becomes tight it can put pressure on the sciatic nerve causing irritation and pain down the back of the leg (sciatica). Peripheral neuritis of the sciatic nerve caused by pressure of an injured or irritated piriformis muscle or an abnormal condition of the piriformis muscle led to piriformis syndrome ${ }^{17}$. In most cases of piriformis syndrome due to contracted piriformis muscle, the sacrum anteriorly rotated toward the ipsilateral side on a contralateral oblique axis, resulting in compensatory rotation of the lower lumbar vertebrae in the opposite direction, this facilitation and compensatory somatic dysfunctions may lead to low back pain, thoracic and cervical pain.

Janda V. ${ }^{19}$ suggested that spasm of the piriformis pulls on the sacrotuberous ligament, causing SI joint pain. Previous studies have indicated that people prefer sitting with the legs crossed because it change the weight or load on soft tissues and muscles; and provides stability to the lower extremities or because of social situations or for aesthetic reasons.

Due to less data in the literature, it was hard to find any previous study which was directly showing the prevalence of piriformis muscle tightness but one study by Singh US et $\mathrm{al}^{20}$ could be cited in previous literature which showed the prevalence of piriformis syndrome as $6.25 \%$ in low back pain patients which is mainly caused by the piriformis tightness as mentioned above.

The piriformis muscle is considered as a postural muscle and the postural muscles has the tendency to become overactive, hypertonic, weak and shortened in length. The piriformis muscle gets overactive and hypertonic during prolonged sitting and walking. When there is a weakness of the primary muscle which is responsible for specific joint movement causes the synergistic muscle to overcome and behave as a primary muscle. Primary work of piriformis is external rotation of the hip and it work as a synergistic muscle to gluteus muscle during hip abduction.

Currently, people spend more time sitting than standing due to the development of science and technology and automation. Dependence on automobiles, even for traveling short distances and long periods spent working on computers; contribute to today's sedentary lifestyles. According to Nicholson $S$ the gluteus muscles are the most neglected group of muscle in whole body as individuals spend their most of the time in sitting and walking very little on the track. So, this may lead to the weakness of gluteal muscle group and synergistic activation of piriformis muscle. This over activation may cause the hypertonicity as well as the tightness of piriformis muscle. Another reason for the over activation of piriformis muscle during a maintained posture is the small size of its muscle fibers. According to Hennemans size principle, the smaller the fiber the easier it activates, the 
larger the fiber the faster it conducts $^{24}$. That's why postural muscles have abundant red fibers. The neurons are smaller and easier to activate, constantly sending impulses with correction messages to the postural muscles to maintain body position. Prolonged activation of muscle may lead to localized muscle tension, muscle strains, muscle fatigue, and other soft-tissue damage. These reasons clearly explain the high prevalence of piriformis muscle tightness in pregnant women

Park Y et $\mathrm{al}^{21}$ concluded the subjects with the habit of sitting with their legs crossed for at least three hours per day had statistically significant differences in the heights of the acromion processes and the ASISs between the two sides.

Dieterich et $\mathrm{al}^{22}$ reported that maximal-effort internal rotation torque in healthy persons increased by about $50 \%$ with the hip flexed as a compared to extend. This increased internal rotation torque with flexion may be due to the increased leverage of some of the internal rotator muscles such as the anterior fibers of the gluteus medius, as depicted in, but also to a reversal of rotary action of some of the traditional external rotators, such as the piriformis, or posterior fibers of the gluteus medius.

Snijders et $\mathrm{al}^{23}$ in their study have shown that cross-legged sitting, which combines flexion and external rotation of the hip, increases the length of the piriformis by $21 \%$ as compared to its length in upright standing.

Data of 150 pregnant female from MGM hospital Aurangabad had been collected out of which 93 female's data had been selected, 57 participants were excluded from the study due to exclusion criteria. In this study, total 93 pregnant women subjects were included, among them 27 subjects were identified as having piriformis muscles tightness, the prevalence of piriformis tightness thus was $29 \%$.

The subjects who participated in this study were pregnant female of third trimester from 18 to 30 years of age. In this study, the number of female subjects $(n=93)$. Out of total affected subjects $(n=27)$, who were presented with piriformis tightness which was clinically diagnosed by piriformis test. There was statistically significant difference of piriformis tightness found in female.

\section{CONCLUSION}

Prevalence of piriformis muscle tightness in pregnant women of third trimester was found to be $29 \%$ in this study which concludes that females who were in third trimester of pregnancy not having piriformis tightness because of relaxin hormone which relaxes all the muscle for the normal growth of fetus.

\section{Acknowledgement: None}

\section{Conflict of Interest: None}

\section{Source of Funding: None}

\section{Ethical Approval: Approved}

\section{REFERENCES}

1. Iliodromitis K, Kociszewski J, Bogossian H. Atrial fibrillation during pregnancy: a 9month period with limited options. Herzschrittmachertherapie+ Elektrophysiologie. 2021 Apr 6:1-6.

2. Mohamed NE. G, Amro DA. Changes in Skeletal System during Pregnancy. Int Gyn \& Women's Health 2 (1)-2018. IGWHC. MS. ID.;127.

3. Datta, D C.textbook of gynecology . s.l. : Jaydeep Brothers Publication LTD , 2014.

4. Sivrioglu AK, Ozyurek S, Mutlu H, Sonmez G. Piriformis syndrome occurring after pregnancy. Case Reports. 2013 Mar 26;2013:bcr2013008946.

5. Katonis P, Kampouroglou A, Aggelopoulos A, Kakavelakis K, Lykoudis S, Makrigiannakis A, Alpantaki K. Pregnancyrelated low back pain. Hippokratia. 2011 $\mathrm{Jul} ; 15(3): 205$.

6. Gomes MR, Araújo RC, Lima AS, Pitangui AC. Gestacional Low Back Pain: Prevalence and Clinical Presentations in a Group of Pregnant Women. Revista Dor. 2013;14:114-7. 
7. Mondal M, Sarkar B, Alam S, Das S, Malik K, Kumar P, Sahay P. Prevalence of piriformis tightness in healthy sedentary individuals: a cross-sectional study. IJHSR. $2017 \mathrm{Jul} ; 7(7): 134-42$.

8. Stewart JD. The piriformis syndrome is overdiagnosed. Muscle \& nerve. 2003 Nov;28(5):644-6.

9. Fishman LM, Dombi GW, Michaelsen C, Ringel S, Rozbruch J, Rosner B, Weber C. Piriformis syndrome: diagnosis, treatment, and outcome - a 10-year study. Archives of physical medicine and rehabilitation. 2002 Mar 1;83(3):295-301.

10. Orthopedic Physical Assessment, Devid J. Magee. Page No, 695.

11. Sencan S, Ozcan-Eksi EE, Cuce I, Guzel S, Erdem B. Pregnancy-related low back pain in women in Turkey: prevalence and risk factors. Annals of physical and rehabilitation medicine. 2018 Jan 1;61(1):33-7.

12. Martin HD, Kivlan BR, Palmer IJ, Martin RL. Diagnostic accuracy of clinical tests for sciatic nerve entrapment in the gluteal region. Knee Surgery, Sports Traumatology, Arthroscopy. 2014 Apr;22(4):882-8.

13. Fligg DB. Biomechanical and treatment considerations for the pregnant patient.The Journal of the Canadian Chiropractic Association. 1986 Sep;30(3):145.

14. Magee, David J. Orthopedic Physical Assessment. 2014.

15. John Ebnezar, Rakesh John. Essentials of orthopedics for physiotherapists. 2012.

16. Maynard F M; Post-Polio Health Newsletter, Vol.28, No.1, 2012.

17. Lynette LY L, Seubsman S. Thai SF-36 health survey: tests of data quality, scaling assumptions, reliability and validity in healthy men and women. Health and Quality of life outcomes.2008;6(52)

18. Chen CK, Nizar AJ. Prevalence of Piriformis Syndrome in Chronic Low Back Pain Patients. A Clinical Diagnosis with
Modified FAIR Test. Pain practice. World Institute of pain. 2012 Aug 2

19. Janda V. Muscle strength in relation to muscle length, pain and muscle imbalance. International Perspectives in physical Therapy 8. Churchill Livingstone: Edinburgh, London, Madrid, Melbourne, New York and Tokyo. 1993: 83-91. In Janda Compendium Volume II.Distributed by OPTP, Minneapolis, MN.undated.

20. Singh US, Meena RK, Singh CAK, Singh A K J, Singh AM, Langshong R; Prevalence of piriformis syndrome among the cases of low back/buttock pain with sciatica: A prospective study. Journal of Medical Society .2013; 27(2);94-99.

21. Park Y, Bae Y. Comparison of Postures According to Sitting Time with the Leg Crossed. J. Phys. Ther. Sci. 2014; 26 (11); 1749-1752

22. A. Dieterich, F. Petzke, C. Pickard, P. Davey, D. Falla. Differentiation of gluteus medius and minimus activity in weight bearing and non-weight bearing exercises by M-mode ultrasound imaging. Manual Therapy 2015; 20(5):715-722.

23. Snijders C J, Hermans P F, Kleinrensink G J. Functional aspects of cross-legged sitting with special attention to piriformis muscles and sacroiliac joints. Clin Biomech (Bristol, Avon). 2006;21: 116-121. http://dx.doi.org/10.1016/j.clinbiomech.200 5.09 .002

24. Henneman E, SomjenG, Carpenter D O. Functional significance of cell size in spinal motoneurons. J. Neurophysiol. 1965;28: 560-80

How to cite this article: Rathod P, Dhawale P, Katage G. Assessment of piriformis tightness in third trimester of pregnancy- a prevalence study. Int J Health Sci Res. 2022; 12(3):61-68. DOI: https://doi.org/10.52403/ijhsr.20220309 